\title{
MENINGITIS BACTERIANA AGUDA
}

\section{ACUTE BACTERIAL MENINGITIS}

DR. RODRIGO BLAMEY D. (1)

1. Unidad de Infectología. Clínica Las Condes.

Email: rblamey.cl

\section{RESUMEN}

La Meningitis Bacteriana Aguda (MBA) de adquisición comunitaria es una enfermedad prevalente en todo el mundo; constituye siempre una emergencia médica y se asocia a una alta morbimortalidad. Su epidemiología es variable y los principales agentes en adultos son S.pneumoniae, N. meningitidis, $H$. influenzae y L. monocitogenes. En Chile existe un sistema de vigilancia recientemente implementado que permitirá un mejor diagnóstico epidemiológico. Las manifestaciones clínicas clásicas no siempre están presentes principalmente en adultos mayores. El diagnóstico requiere del estudio de líquido cefalorraquídeo, y las técnicas de biología molecular han significado un aporte relevante en los últimos años. El tratamiento antibiótico debe ser instaurado rápidamente para mejorar el pronóstico, mientras que la terapia coadyuvante con corticoides en adultos tiene sólo beneficios en etiología neumocócica. Se requieren mejores estrategias de prevención frente a una entidad que no ha cambiado su mortalidad a pesar del progreso de la medicina moderna.

Palabras clave: Meningitis bacteriana, epidemiología, líquido cefalorraquídeo, antibióticos, complicaciones.

\section{SUMMARY}

Community acquired acute bacterial meningitis is a life-threatening disease prevalent worldwide, it evolves with dynamic epidemiology and is associated with a high morbidity and mortality. Main agents involved in adult population are S. pneumoniae, $N$. meningitidis, $H$. influenzae and L. monocitogenes. Recently, a new surveillance system was implemented in Chile to improve epidemiological reports.
Clinical clues are not always present, and diagnosis can be a challenge in elderly. Diagnosis is based in cerebrospinal fluid analysis, where nuclear acid amplification test has improved sensitivity of traditional microbiology tools. Antibiotics are mainstay of therapy and must be administered as soon as possible to improve prognosis. Steroids have a limited role specifically in S. pneumoniae meningitis. Better prevention strategies are required in face to the poor prognosis described despite progress in modern medicine.

Key words: Bacterial meningitis, epidemiology, cerebrospinal fluid, antibiotics, complications.

\section{INTRODUCCIÓN}

La Meningitis Bacteriana Aguda (MBA) corresponde a la inflamación de las meninges por bacterias piógenas. Es una patología prevalente en todo el mundo, que siempre constituye una emergencia médica.

La epidemiología es variable, dependiendo de la edad, del agente y de la presencia de ciertos factores de riesgo en cada paciente. La MBA en adultos, comunitaria, será el objeto de esta revisión.

Los principales agentes descritos corresponden a Streptococcus pneumoniae, Neisseria meningitidis, Haemophilus influenzae y Listeria monocitogenes. La mayoría de los casos son de adquisición comunitaria mientras que otras bacterias como por ejemplo Staphylococcus spp. y bacilos gram negativos pueden provocar meningitis, pero habitualmente en contexto nosocomial (asociada a procedimientos médicos) o secundario a trauma. 
A pesar de los progresos en la medicina intensiva y amplia gama de antibióticos disponibles, la mortalidad de la MBA se ha mantenido estable durante las últimas décadas, lo cual constituye un desafío permanente y refuerza la necesidad de incorporar mejores estrategias de prevención (1).

\section{EPIDEMIOLOGÍA}

La epidemiología de la MBA ha cambiado sustancialmente con la introducción de vacunas efectivas contra algunos de los principales patógenos y así ha sido demostrado en diferentes países. Desde la década de los 90 la introducción de vacunas conjugadas pediátricas contra $\mathrm{H}$. influenzae tipo b, posteriormente para S. pneumoniae y para $N$. meningitidis serogrupo $C$, han hecho disminuir la incidencia de estos agentes, principalmente en los grupos vacunados así como en el resto de la población, debido al "efecto rebaño" que poseen este tipo de vacunas. Cifras recolectadas en Estados Unidos demuestran una reducción del 31\% en la incidencia global de MBA desde 1998 a 2007 (2). Para el período 2003 - 2007, los principales agentes reportados para adultos en este mismo estudio fueron $S$. pneumoniae $(71 \%)$, N. meningitidis $(12 \%)$, S. agalactiae $(7 \%), H$. influenzae $(6 \%)$ y L. monocitogenes (4\%), destacando que la presencia de inmunosupresión se registró en el $22,5 \%$ de los casos y la presencia de enfermedades crónicas en 32,7\% (2). Otros países como España también han reportado cambios epidemiológicos similares, atribuidos entre otros, al uso de vacunas, aumento de edad y de comorbilidades en la población (3).

El panorama global actual describe a S. pneumoniae y N. meningitidis como los agentes de MBA más frecuentes en adultos. S. pneumoniae es el principal agente a nivel global en países europeos y americanos, con una elevada mortalidad asociada, de entre 16 y $33 \%(5,6)$. Actualmente se han descrito 92 serotipos diferentes de $S$. pneumoniae de acuerdo al polisacárido capsular (7), pero sólo unos pocos de ellos son responsables de la mayoría de infecciones invasoras. Los serotipos de $S$. pneumoniae implicados en meningitis en adultos han variado en Estados Unidos después de la introducción de la vacuna heptavalente, con un incremento de los serotipos no vacunales. Se esperan nuevos cambios epidemiológicos con la reciente introducción de la vacuna con 13 serotipos (2). El mayor riesgo para enfermedad neumocócica invasora lo presentan pacientes mayores de 65 años, comorbilidades como insuficiencia cardíaca, enfermedades pulmonares, renales y hepáticas crónicas, diabetes mellitus, tabaquismo, alcoholismo e inmunodeficiencias tales como infección por VIH, mieloma múltiple, neoplasias, uso de inmunosupresores, esplenectomizados o con asplenia funcional, portadores de implantes cocleares y fistulas de LCR (8).

N. meningitidis se ha clasificado en 13 serogrupos; seis de los cuales son los principales causales de enfermedad ( $A, B, C, X, W-135$ e Y) (9). Este agente se presenta con un patrón endémico y epidémico sin embargo, su epidemiología es variable e impredecible. La mayoría de países en América y Europa presentan baja endemia con epidemias esporádicas principalmente por serogrupos B y C, mientras que en Áfri- ca subsahariana existe una alta endemia, con epidemias producidas por diferentes serogrupos como A, C y W-135, en lo que se conoce como el cinturón meningítico. Desde el año 2000 se ha producido una diseminación mundial de un clon W-135, hipervirulento, originalmente descrito en la peregrinación a La Meca, y que ha sido responsable de brotes en diferentes latitudes, incluyendo países sudamericanos como Argentina, Uruguay, Brasil y Chile $(10,11)$. Los factores de riesgo para enfermedad meningocócica incluyen a menores de un año, tabaquismo, contacto cercano con casos, inmunodeficiencias como esplenectomizados o con asplenia funcional, alteraciones del sistema del complemento, exposición ocupacional (laboratorios de microbiología) y viajeros a zonas de riesgo $(12,13)$.

En relación a L. monocitogenes, se han observado casos principalmente en brotes con mayor riesgo en embarazadas, neonatos y mayores de 60 años. Otros factores de riesgo son pacientes alcohólicos, diabéticos, oncológicos, usuarios de corticoides e inmunosupresores, usuarios de biológicos bloqueadores de TNF-a, nefrópatas, enfermedades hepáticas crónicas y estados de sobrecarga de hierro. Se han descrito brotes en relación a alimentos contaminados (vegetales, lácteos y carnes) (6).

En Chile no existía vigilancia de MBA hasta diciembre de 2011, año en que se inicia la vigilancia epidemiológica obligatoria por parte del Ministerio de Salud y cuyo organismo responsable es el Instituto de Salud Pública de Chile (ISP) (14). Sin embargo, los datos de esta vigilancia aún no han sido publicados. Hasta la fecha sólo existen datos publicados por separado de la vigilancia de enfermedad meningocócica y de la vigilancia de laboratorio de enfermedad invasora por $S$. pneumoniae y por $\mathrm{H}$. influenzae, por lo que no existe información confiable para MBA como tal.

En relación a la enfermedad meningocócica, existe en el país una baja endemia, con una tasa de incidencia en disminución durante la última década, hasta alcanzar 0,4/100.000 hab. en 2011. Han habido brotes esporádicos y acotados a ciertas ciudades por serogrupos $B$ y $C$ en los años 1980, 1993 y 2000. Desde 2011, hemos presenciado un cambio epidemiológico con aumento de la incidencia por sobre las cifras endémicas habituales, con un cambio en los serogrupos predominantes de N. meningitidis, ya que históricamente el serogrupo B fue el más importante (más del $60 \%$ de los casos), y ha sido desplazado por la emergencia del serogrupo W-135, que hasta 2013 alcanzó al 62,5\% de los casos. Sin embargo, del total de 136 casos de enfermedad meningocócica, aproximadamente un tercio se registró como meningitis. Es interesante señalar que la incorporación de técnicas de biología molecular permitió realizar diagnóstico de N. meningitidis en LCR en 3.9\% de las muestras con cultivo negativo (18/458) (15).

Los datos de vigilancia de enfermedad invasora por S. pneumoniae demostraron que para el período 2007 - junio 2013, sólo el 10,3\% de las 5.131 muestras estudiadas provenía de líquido cefalorraquídeo, implicando unos 80 a 100 casos anuales, lo que probablemente es un 
subregistro que debiera corregirse con los datos del nuevo sistema de vigilancia de MBA (27).

Por último, la vigilancia de enfermedad invasora por $\mathrm{H}$. influenzae registró para el período 2007 - junio 2012, un total de 68 cepas (incluyendo pediátricas y de adultos), de las cuales el $62 \%$ correspondieron a niños menores de cinco años, por lo que este agente no representa un problema de relevancia en MBA de adultos en Chile (28).

\section{FISIOPATOLOGÍA}

Habitualmente los agentes causales de MBA colonizan el epitelio nasofaríngeo e ingresan al sistema nervioso central por vía hematógena. La colonización epitelial es facilitada por diferentes mecanismos como son la lesión epitelial producida por infecciones respiratorias virales, el tabaquismo y por la producción de proteasas que destruyen la lg A local (16). Sin embargo, la colonización no es suficiente para provocar infección y es así que por ejemplo, N. meningitidis es considerado principalmente un comensal del epitelio nasofaríngeo humano, siendo una incertidumbre los factores precisos que determinan la aparición de la enfermedad meningocócica.

Una vez en el torrente sanguíneo, la presencia de cápsula polisacárida es fundamental para evadir la fagocitosis y la lisis mediada por complemento, y es un factor de virulencia presente en $S$. pneumoniae, $N$. meningitidis y $\mathrm{H}$. influenzae. Si bien muchas bacterias son capaces de producir bacteriemia, sólo un número muy reducido de ellas tiene la capacidad de provocar meningitis. La penetración de la barrera hemato-encefálica por los diferentes patógenos ocurre por mecanismos hasta ahora no bien dilucidados sin embargo, se ha descrito que los tres principales patógenos utilizan la molécula de Receptor de Laminina (37 kDa) como receptor común para unirse al endotelio vascular cerebral (barrera hematoencefálica) mediante adhesinas de superficie (Cbpa en S. pneumoniae, PilQ y PorA en N. meningitidis, y OmpP2 en H. influenzae), y posteriormente mediante la unión de fosforilcolinas de la pared, se unirían al receptor del Factor Activador de Plaquetas (PAFr), activando un proceso de endocitosis mediada por $\beta$-arrestina, por supuesto mimetismo molecular con el PAF $(4,17)$.

Una vez ocurrido este proceso, pueden multiplicarse en ausencia de leucocitos y otros componentes del sistema inmune, características del líquido cefalorraquídeo (LCR) normal. Una vez invadido el LCR, se gatilla la cascada inflamatoria con liberación de citoquinas, quimiotaxis de leucocitos y aumento de permeabilidad vascular, que finalmente determinan el grado de inflamación meníngea, edema cerebral y daño neuronal, todos elementos relacionados con la morbimortalidad asociada a la MBA $(4,6)$. Es importante destacar el rol que poseen algunos componentes estructurales bacterianos, como ejemplo en S. pneumoniae, en gatillar los procesos inflamatorios en modelos experimentales de MBA, ya que esto ha permitido conocer que una parte de la injuria tisular producida es mediada por la acción bacteriolítica de los antimicrobianos y por la vigorosa respuesta immune del huésped y por lo tanto, podrían tener un rol relevante las terapias inmunomoduladoras (18).

\section{Aspectos clínicos}

La tríada clásica de la MBA comprende fiebre, cefalea y signos de irritación meníngea, a lo cual pueden agregarse signos de disfunción cerebral como confusión y alteración del nivel de conciencia. La cefalea es holocránea, y diferente a un cuadro de cefalea habitual. La frecuencia de estos signos y síntomas es variable, por lo que la ausencia de alguno de ellos no descarta la posibilidad de MBA. Además ocurren con menor frecuencia náuseas, vómitos, convulsiones y signos de déficit neurológicos que pueden traducir fenómenos isquémicos. Finalmente pueden aparecer los signos de hipertensión endocraneana como hipertensión arterial, bradicardia y coma (6).

Algunos datos semiológicos pueden orientar a la etiología, como la presencia de rash, artritis o la rápida instalación del shock en el caso de la meningococcemia, o la presencia de ataxia, compromiso de pares craneales y nistagmus que pueden traducir una romboencefalitis por L. monocytogenes (6).

En adultos mayores la sospecha puede ser más difícil ya que la fiebre y los signos meníngeos pueden estar ausentes, debiendo sospechar el diagnóstico por el compromiso de conciencia. La frecuencia de discopatías y otras alteraciones en la columna cervical hacen difícil de pesquisar los signos de irritación meníngea y habitualmente el diagnóstico es más tardío asociándose a mayor mortalidad (16). Muchas veces el antecedente de infección ótica, sinusal o respiratoria previa puede hacer sospechar la etiología neumocócica.

La coexistencia poco habitual de MBA, neumonía y endocarditis debe hacer sospechar etiología neumocócica, configurando el Síndrome de Austrian, cuyo diagnóstico habitualmente es tardío (19).

\section{Complicaciones y pronóstico}

Las complicaciones suelen ocurrir precozmente en la evolución de las MBA. Las más frecuentes son la sordera sensorioneural y la disfunción vestibular, que ocurren más frecuentemente asociadas a S. pneumoniae, con frecuencias del 14 al $54 \%$, muchas veces no documentadas sino por audiometría (23).

Otras complicaciones más serias corresponden al edema cerebral con hidrocefalia y complicaciones vasculares como trombosis de senos venosos e infartos cerebrales. Los déficit neurológicos resultantes, principalmente motores, visuales y afasia, se describen en rango del 20 al $50 \%$ en las distintas series.

Complicaciones menos frecuentes que ocurren, comprenden colecciones subdurales infectadas (empiema) o estériles (higromas), abscesos cerebrales y ventriculitis.

Las secuelas a nivel neurocognitivo (deterioro intelectual) pueden ser más sutiles pero están descritas a largo plazo, afectando una propor- 
ción importante de pacientes (32\%), al igual que otras complicaciones, especialmente con MBA por S. pneumoniae.

La mortalidad de la MBA en adultos es más elevada según la edad del paciente. En estadísticas de Estados Unidos, con una mortalidad global para adultos en años recientes de $16.4 \%$, el grupo de 18 a 34 años tuvo una mortalidad del $8.9 \%$ comparado con el $22.7 \%$ de los mayores de 65 años (2). Varios reportes concuerdan en la mayor mortalidad de la MBA producida por $S$. pneumoniae versus otros agentes.

Se ha establecido un modelo pronóstico en MBA de acuerdo a las características clínicas de ingreso, descrito en un estudio con 176 adultos y validado en otro grupo de 93 pacientes. Destaca la utilización de sólo tres factores clínicos: hipotensión, alteración del nivel de conciencia y convulsiones, los cuales fueron asociados con evolución desfavorable (mortalidad intrahospitalaria o secuela neurológica al alta) según el siguiente modelo (24):

1. Bajo riesgo (ausencia de factores): $9 \%$ evolución desfavorable

2. Riesgo intermedio (presencia de un factor): $33 \%$ evolución desfavorable

3. Alto riesgo (presencia de dos o tres factores): $56 \%$ evolución desfavorable

\section{DIAGNÓSTICO}

El diagnóstico de MBA se basa en la demostración de infección piógena en el LCR y por lo tanto, se debe estudiar el LCR mediante punción lumbar, para identificar el agente etiológico y conocer la susceptibilidad a los antibióticos (antibiograma).

Aunque no se mide habitualmente en nuestro medio, la presión del LCR se describe aumentada en casi todos los casos. La pleocitosis

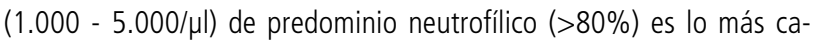
racterístico, sin embargo, se describen recuentos normales o bajos en algunos casos, 0 a veces un predominio inicial de linfocitos. Un recuento bajo de leucocitos en presencia de gran cantidad de bacterias se asocia a mal pronóstico. La hipoglucorraquia menor a $40 \mathrm{mg} / \mathrm{dL}$ se ha descrito en $60 \%$ de los casos, y una relación LCR/sangre menor a 0.31 en un $70 \%$. Otro elemento sugerente es la concentración elevada de proteínas (100 - $500 \mathrm{mg} / \mathrm{dL}$ ), observada en la mayoría de los casos y una concentración de lactato mayor de $35 \mathrm{mg} / \mathrm{dL}$ (6).

La tinción de gram es un método fácil y rápido que permite visualizar las bacterias en el LCR en 60 a 90\% de los casos, con una especificidad del $100 \%$. Su positividad se correlaciona con la concentración bacteriana, ya que cifras de $1 \times 10^{3} \mathrm{UFC} / \mathrm{ml}$ se relacionan a un $25 \%$ de observaciones positivas, mientras que recuentos de más de $1 \times 10^{5}$ otorgan alrededor de $97 \%$ de positividad (20). Las morfologías bacterianas que son sugerentes corresponden a diplococos gram positivos (S. pneumoniae), diplococos gram negativos (N. meningitidis) y bacilos gram positivos (L. monocitogenes).
El cultivo es positivo entre el 70 al $85 \%$ de los pacientes. Se debe considerar que el rendimiento de la tinción de gram y del cultivo puede ser notablemente menor en pacientes que hayan recibido antibióticos previamente. Se debe recalcar también que la fácil adquisición de antibióticos por la población general, la frecuente prescripción de antibióticos sin diagnóstico definido en los servicios de emergencias y la variabilidad de los recursos de diagnóstico microbiológico en los distintos centros médicos del país, hacen que un porcentaje importante de MBA queden sin diagnóstico etiológico en nuestro medio.

La detección de antígenos bacterianos solubles en LCR mediante técnica de látex ha mejorado parcialmente la identificación de agentes bacterianos en MBA. Esta técnica puede detectar antígenos de S. pneumoniae, N. meningitidis, $H$. influenzae tipo b, E. coli y S. agalactiae. El impacto clínico ha sido menor del esperado y por lo tanto, no se recomienda de rutina $(6,20)$.

El método que más ha contribuido al rendimiento diagnóstico del LCR en los últimos años ha sido la biología molecular. Se puede amplificar ácidos nucleicos para detección de cualquier bacteria, sin embargo, estudios con partidores para los agentes más frecuentes (S. pneumoniae, $N$. meningitidis, $H$. influenzae tipo $b$ ), han mostrado excelentes valores de sensibilidad (100\%) y valor predictivo negativo (mayor a 99\%). En la experiencia de Brasil, con un promedio anual de 28.000 casos, se incorporó una técnica de PCR de tiempo real en la ciudad de Sao Paulo, con sensibilidades para S. pneumoniae, N. meningitidis, H. influenzae tipo b, del $97.8 \%, 100 \%$ y $66.7 \%$ respectivamente, y especificidades del 98 al 100\%. Esto significó un aumento del 52\% en la detección para S. pneumoniae, de un $85 \%$ para N. meningitidis y de un $20 \%$ para $H$. influenzae tipo b, respecto a los métodos microbiológicos tradicionales (21). En nuestro país se ha implementado en algunos centros hospitalarios y en el ISP para la vigilancia epidemiológica.

En cuanto a imágenes, se recomienda la realización de TAC cerebral previo a la realización de punción lumbar para disminuir el riesgo de herniación cerebral, sólo en pacientes que posean alguna de las siguientes condiciones (20):

1- Inmunosupresión

2- Antecedentes de lesiones cerebrales

3- Convulsiones de inicio reciente

4- Edema de papila

5- Compromiso de conciencia

6- Déficit neurológico focal

Aunque no existen contraindicaciones absolutas para realizar una punción lumbar, se debe tener especial precaución cuando existe aumento de presión intracraneana (PIC), coagulopatía y sospecha de absceso epidural (22).

Siempre se deben tomar exámenes generales para cuantificar el síndrome inflamatorio y detectar disfunción de sistemas (recuentos hemato- 
lógicos, coagulación, función renal, hepática, gases arteriales, lactato) y dos frascos de hemocultivos (de venopunciones diferentes), previo al inicio de terapia antibiótica, ante la posibilidad de retardo del estudio de LCR por diversos motivos.

\section{TRATAMIENTO}

Una vez sospechado el diagnóstico de MBA se debe hospitalizar al paciente en una unidad con monitoreo (unidad de cuidados intermedios), realizar los exámenes diagnósticos e iniciar el tratamiento antibiótico en forma inmediata. En caso que exista retraso en la posibilidad de realizar el estudio del LCR, se deben tomar los exámenes de sangre antes mencionados e iniciar tratamiento antibiótico en base a las características clínico-epidemiológicas del paciente. Esto se debe a que diversos estudios han demostrado un peor pronóstico, con mayor incidencia de secuelas neurológicas y mayor mortalidad en caso de retraso en el inicio de terapia antibiótica $(6,20)$. En caso de una presentación clínica con criterios de disfunción orgánica múltiple, el paciente debe ser estabilizado y tratado en una unidad de cuidados intensivos, con eventual instalación de monitorización de la PIC y maniobras para bajar la PIC (ej. hiperventilación).

La recomendación de antibióticos se hace de acuerdo al diagnóstico etiológico presuntivo, sin tener aún los resultados de cultivos. Si hay sospecha etiológica por factores clínicos o en la tinción de gram para S. pneumoniae, $\mathrm{N}$. meningitidis o $\mathrm{H}$. influenzae, se debe iniciar trata- miento con ceftriaxona $2 \mathrm{~g}$. cada 12 hrs. iv. Si hay sospecha de listeriosis, se debe agregar ampicilina $2 \mathrm{~g}$. $\mathrm{c} / 4 \mathrm{hrs}$. iv.

Una vez determinado el agente etiológico y la susceptibilidad, se puede adecuar el tratamiento antibiótico de acuerdo a lo indicado en la tabla 1 (20).

En relación a los principales agentes etiológicos de MBA en el país, la vigilancia del ISP ha reportado para las cepas de S. pneumoniae (período 2007-2013) un 78\% de susceptibilidad a penicilina y $98 \%$ a cefotaxima en el grupo de mayores de 15 años (27), mientras que para N. meningitidis (período 2013) hubo un $61 \%$ de susceptibilidad a penicilina y $100 \%$ para cefotaxima, ciprofloxacino, cloramfenicol y rifampicina (15). Se debe recordar que los puntos de corte en S. pneumoniae para la interpretación de susceptibilidad a penicilina corresponden a cepas de foco meníngeo, que son diferentes a los utilizados en cepas de focos extrameníngeos, donde los porcentajes de susceptibilidad son mayores (29).

El uso de corticoides como terapia adyuvante se ha utilizado por largo tiempo en modelos experimentales, mostrando beneficios en la reducción de la inflamación del territorio cerebral. Sin embargo, en estudios clínicos, sólo ha mostrado su utilidad en MBA de adultos por S. pneumoniae, donde ha disminuido la mortalidad y las secuelas neurológicas. En dicho contexto se recomienda usar dexametasona $10 \mathrm{mg}$ cada 6 hrs. iv. durante cuatro días (25). Cabe señalar que los estudios que han demostrado beneficios fueron todos realizados en países desarro-

\section{TABLA 1 TRATAMIENTO ANTIBIÓTICO DE LA MBA}

\begin{tabular}{|c|c|c|}
\hline AGENTE ETIOLÓGICO & TERAPIA ELECCIÓN & TERAPIA ALTERNATIVA \\
\hline $\begin{array}{l}\text { S. pneumoniae } \\
\text { - CIM PNC }<0.06 \\
\text { - CIM PNC } \geq 0.12 \\
\text { + } \text { CIM ceftriaxona }<1.0 \\
\text { + CIM ceftriaxona } \geq 1.0\end{array}$ & $\begin{array}{l}\text { Penicilina sódica, ampicilina } \\
\text { Ceftriaxona } \\
\text { Vancomicina + ceftriaxona }\end{array}$ & $\begin{array}{l}\text { Ceftriaxona, Cloranfenicol } \\
\text { Cefepime, Meropenem } \\
\text { Moxifloxacino }\end{array}$ \\
\hline $\begin{array}{l}\text { N. meningitidis } \\
\text { - CIM PNC <0.1 } \\
\text { - CIM PNC } 0.1-1.0\end{array}$ & $\begin{array}{l}\text { Penicilina sódica, ampicilina } \\
\text { Ceftriaxona }\end{array}$ & $\begin{array}{l}\text { Ceftriaxona, cloranfenicol } \\
\text { Cloranfenicol, fluorquinolonas, meropenem }\end{array}$ \\
\hline $\begin{array}{l}\text { H. influenzae } \\
\text { - } \beta \text { lactamasa negativo } \\
\text { - } \beta \text { lactamasa positivo }\end{array}$ & $\begin{array}{l}\text { Ampicilina } \\
\text { Ceftriaxona }\end{array}$ & $\begin{array}{l}\text { Ceftriaxona, cloranfenicol, fluorquinolonas } \\
\text { Cefepime, cloranfenicol, fluorquinolonas }\end{array}$ \\
\hline L. monocitogenes & Ampicilina, PNC sódica & Cotrimoxazol, meropenem \\
\hline S. agalactiae & Ampicilina, PNC sódica & Ceftriaxona, Vancomicina \\
\hline
\end{tabular}

CIM: Concentración inhibitoria mínima, PNC: Penicilina.

Modificado de la ref. 20 y 29. 
llados, en cambio, otros estudios realizados en países subdesarrollados (como Malawi y Vietnam) no han replicado los mismos resultados, aunque el contexto epidemiológico ha sido distinto. Datos concordantes fueron publicados en un meta análisis reciente, donde se concluye que los corticoides demostraron una menor mortalidad sólo en MBA por S. pneumoniae, pero no en $N$. meningitidis ni $H$. influenzae, ni se disminuyó la mortalidad global. Además, se demostró una reducción de secuelas neurológicas sólo en países desarrollados, pero no en los subdesarrollados (26). Los beneficios de su uso se observan solamente si se administra 20 minutos antes o concomitante a los antibióticos, pero no tiene utilidad en forma posterior al inicio de la antibióticoterapia.

Si la evolución del paciente es favorable, se completarán los plazos dependiendo de cada agente etiológico (10 - 14 días para S. pneumoniae, 7 - 10 días para N. meningitidis, 21 o más días para L. monocitogenes), según la evolución clínica y si existen o no otros focos clínicos (endocarditis, artritis, etc.). Si la evolución no es favorable, debe realizarse un control del LCR a los dos días y agregar la búsqueda de complicaciones u otros focos que puedan determinar el fracaso del tratamiento (ej. abscesos).

\section{PREVENCIÓN}

Dada la elevada tasa de morbimortalidad de la MBA, que se ha mantenido estable en las últimas décadas, se hace imperioso contar con mejores estrategias de prevención.

En relación a la prevención de casos secundarios, se recomienda administrar profilaxis antibiótica en el caso de pacientes con MBA por $N$. meningitidis, a todos los contactos cercanos (convivencia por más de 8 hrs. 0 expuesto a secreciones del caso índice durante la última semana previa al inicio de síntomas y hasta 24 hrs. post inicio de terapia antibiótica), pudiendo utilizar ciprofloxacino $500 \mathrm{mg}$. dosis única, ceftriaxona $250 \mathrm{mg}$. im. dosis única o rifampicina $600 \mathrm{mg}$. cada 12 hrs. vo. durante 2 días (6).

La herramienta más útil en la prevención de MBA han sido las vacunas. Estas poderosas herramientas han cambiado la epidemiología de la MBA en aquellos países que las han introducido. Sin embargo, tanto S. pneumoniae como N. meningitidis poseen la capacidad de cambio fenotípico capsular que ha significado el reemplazo de las cepas circulantes por variantes no incluidas en las vacunas utilizadas. Este tema será abordado en otra sección de este mismo número.

\section{REFERENCIAS BIBLIOGRÁFICAS}

1. Swartz M. Bacterial meningitis - A view of the past 90 years N Eng J Med 2004;351(18):1826-8.

2. Thigpen MC. et al. Bacterial meningitis in the United States 1998 - 2007 N Eng J Med 2011;364:2016-25.

3. Domingo $P$. et al. The changing pattern of bacterial meningitis in adult patients at a large tertiary university hospital in Barcelona, Spain (1982-2010) J Infection 2013;66:147-54.

4. Nudelman Y, Tunkel A. Bacterial meningitis: Epidemiology, pathogenesis and management update Drugs 2009;69(18):2577-96.

5. Kastenbauer S, Pfister HW. Pneumococcal meningitis in adults, Spectrum of complications and prognostic factors in a series of 87 cases Brain 2003;126:101525.

6. Tunkel A. et al. Acute Meningitis, en Mandell, Douglas and Bennett's Principles and Practice of Infectious Diseases, 2010, 7th Edition, Churchill - Livingstone.

7. Cálix J, Nahm M. A New Pneumococcal Serotype, $11 \mathrm{E}$, Has a Variably Inactivated wcjE Gene J Infect Dis 2010;202(1):29-38.

8. Sexton D, Invasive pneumococcal (Streptococcus pneumoniae) infections and bacteremia en www.uptodate.com, acceso 01 marzo 2014.

9. Chang Q. et al. Meningococcal disease: Changes in epidemiology and prevention Clin Epidemiol 2012;4:237-45.

10. Harrison L. Prospects for Vaccine Prevention of Meningococcal Infection Clin Microbiol Rev 2006;19(1):142-64.

11. López E, Debagg R. Enfermedad meningocóccica: siempre presente. Cambios en los serogrupos en el Cono Sur Rev Chilena Infectol 2012;29(6):587-94.
12. Apicella M. Epidemiology of Neisseria meningitidis infection, en www. uptodate.com, acceso 01 marzo 2014.

13. Cohn AC. et al., Prevention and control of meningococcal disease: recommendations of the Advisory Committee on Immunization Practices (ACIP) MMWR 2013; 62(RR-2):1-28.

14. Circular B51/50 Circular de vigilancia epidemiológica de meningitis bacteriana Departamento de epidemiología, MINSAL, Chile, 5 diciembre 2011.

15. Instituto de Salud Pública de Chile, Informe de Resultados de Vigilancia de Laboratorio Enfermedad Invasora Neisseria meningitidis 2013.

16. Mace S, Acute bacterial meningitis Emerg Med Clin N Am 2008;38:281-317.

17. Orihuela C. et al. Laminin receptor initiates bacterial contact with the blood brain barrier in experimental meningitis models J Clin Invest 2009; 119:1638-46.

18. Henriques-Normark B, Tuomanen E. The Pneumococcus: Epidemiology, Microbiology, and Pathogenesis Cold Spring Harb Perspect Med 2013;3:a010215.

19. Kanakadandi V. et al. The Austrian syndrome: a case report and review of the literature Infection 2013; 41(3):695-700.

20. Tunkel $A$. et al. Practice guidelines for the management of bacterial meningitis Clin Infect Dis 2004; 39:1267-84.

21. Sacchi C. et al. Incorporation of Real-Time PCR into Routine Public Health Surveillance of Culture Negative Bacterial Meningitis in Sao Paulo, Brazil PLoS One 2011; 6(6): e20675.

22. Johnson K, Sexton D. Lumbar puncture: Technique, indications, contraindications, and complications in adults en www.uptodate.com, acceso 03 marzo 2014. 
23. Worsøe L. et al. Factors Associated with the Occurrence of Hearing Loss after Pneumococcal Meningitis Clin Infect Dis 2010; 51 (8): 917 - 924.

24. Aronin S. et al. Community-acquired bacterial meningitis: risk stratification for adverse clinical outcome and effect of antibiotic timing Ann Intern Med. 1998;129(11):862-9.

25. De Gans J, Van de Beek D. Dexamethasone in adults with bacterial meningitis N Eng J Med 2002;347:1549-56.

26. Brouwer $M C$. et al. Corticosteroids for acute bacterial meningitis (Review)"Cochrane Database Syst Rev. 2013 Jun;6:CD004405.

27. Vigilancia de Enfermedad Invasora Streptococcus pneumoniae. Chile, 2007 2013 Boletín Instituto de Salud Pública de Chile, vol. 3, № 8, Julio 2013.

28. Vigilancia de laboratorio de Haemophilus influenzae tipo b. Chile 2007 - 2012 Boletín Instituto de Salud Pública de Chile, vol. 2, № 14, Octubre 2012

29. Weinstein MP. et al. Rationale for revised penicillin susceptibility breakpoints versus Streptococcus pneumoniae: coping with antimicrobial susceptibility in an era of resistance Clin Infect Dis 2009;48(11):1596-600.

El autor declara no tener conflictos de interés, en relación a este artículo. 